PROCEEDINGS OF THE

AMERICAN MATHEMATICAL SOCIETY

Volume 138, Number 9, September 2010, Pages 3241-3256

S 0002-9939(2010)10480-X

Article electronically published on May 13, 2010

\title{
DISCRETE FUNCTION THEORY BASED ON SKEW WEYL RELATIONS
}

\author{
HILDE DE RIDDER, HENNIE DE SCHEPPER, UWE KÄHLER, AND FRANK SOMMEN
}

(Communicated by Michael T. Lacey)

\begin{abstract}
In this paper we construct the main ingredients of a discrete function theory in higher dimensions by means of a new "skew" type of Weyl relations. We will show that this new type overcomes the difficulties of working with standard Weyl relations in the discrete case. A Fischer decomposition, Euler operator, monogenic projection, and basic homogeneous powers will be constructed.
\end{abstract}

\section{INTRODUCTION}

Currently, there seems to be much interest in finding discrete counterparts of various structures in classical (continuous, smooth) mathematics. An important issue is the construction of a discrete function theory, not only as a counterpart of the theory of holomorphic functions in the complex plane, which is nowadays well established, but also of its higher-dimensional version(s). Essentially these higherdimensional analogues were developed in two major directions, the first one being several complex variables analysis and the second one being Clifford analysis, i.e. the theory of null solutions of a Dirac operator, called monogenic functions, [1, 6]. In the present paper, we are investigating a discrete counterpart of the latter. For a discrete version of the case of several complex variables we refer to the literature, e.g. [4].

While there already are a lot of contributions regarding the construction of discrete Dirac operators, see e.g. [19, 12, 16, 9, 11, 15, there still is much less development on the actual discrete function theory in higher dimensions. The existing approaches are either based on potential-theoretical arguments, e.g. [14, 13, 2, 3, or on direct calculations using only one type of difference operator, e.g. [10, but neither one leads to truly satisfactory results. Potential-theoretical arguments allow us to obtain a discrete Cauchy kernel and hence a discrete Cauchy integral formula, involving in the cited papers also the discrete Fourier transform, but do not lead to any results concerning basic polynomials, a Fischer decomposition, Taylor series, etc. The approach of using only one type of difference operator, i.e. either forward or backward differences, on the other hand, allows the construction of basic polynomials and a Fischer decomposition, but it does not cover the case of a Dirac

Received by the editors December 17, 2009.

2010 Mathematics Subject Classification. Primary 39A12, 30G35, 39A70, 06D50.

Key words and phrases. Discrete monogenic polynomial, Fischer decomposition.

(C)2010 American Mathematical Society 
operator factorizing the classic star Laplacian. For such a factorization, both types of difference operators are necessary.

The picture becomes more clear when we take another look at the continuous case, from an abstract point of view. From such a point of view the construction of a function theory follows a similar philosophy as, for instance, umbral calculus: one needs so-called raising and lowering operators, i.e. operators which turn a polynomial of degree $k$ into one of a higher or lower degree. The lowering operator is usually given as a differential operator, while the raising operator takes the form of a multiplication operator. If the lowering operators are given, the raising operators are usually defined by means of so-called "Weyl relations", which for the classical partial derivatives take the form

$$
\partial_{j} x_{j}-x_{j} \partial_{j}=1
$$

or, applied to a function $f$,

$$
\partial_{j}\left(x_{j} f(x)\right)-x_{j} \partial_{j} f(x)=f(x) .
$$

Afterwards, the corresponding theory is constructed based on a duality argument between both types of operators, the so-called Fischer duality.

In the discrete case, however, a major obstacle to this approach arises. While forward and backward differences are mutually commuting with each other, this is no longer true for the corresponding vector variables, whence a direct approach using standard Weyl relations will not enable us to use duality arguments between difference operators and vector variables, since the resulting algebraic structures will be different. To overcome this obstacle, we propose a modified approach. Instead of the standard ones we introduce a type of "skew" Weyl relations, the underlying idea being that, using the standard Weyl relations, forward and backward difference operators and their corresponding vector variables are treated as being completely independent and, in this way, we accept having to deal with the same (difficult) problems which appear in the case of several Dirac operators. But, while this is tacitly accepted in the stated papers, it is by no means necessary; i.e. in the resulting discrete function theory there is no real independence. We will show that our modified approach will allow us to construct all necessary elements for a higherdimensional function theory. In Section 3 we construct the Euler operator in this setting, in order to define homogeneous polynomials, and we show that the result of the action of the new vector variable on a classical homogeneous power can be given in terms of Euler polynomials of even degree. In Section 4 we establish a Fischer decomposition, the related monogenic projection, as well as the dimension of the spaces of discrete monogenic, homogeneous polynomials.

\section{Preliminaries}

For simplicity we restrict ourselves to the grid $\mathbb{Z}^{m}$ with orthogonal basis $\mathbf{e}_{k}$, $k=1, \ldots, m$. The forward and backward differences $\Delta_{j}^{ \pm}$are traditionally given by

$$
\Delta_{j}^{ \pm} u=\mp\left(I-\sigma_{h}^{ \pm j}\right) u,
$$

where $I$ denotes the identity operator and $\sigma_{h}^{ \pm j} u=u\left(\cdot \pm h \mathbf{e}_{j}\right)$ denotes the shift operator on the grid $\mathbb{Z}^{m}$, which, in the standard case considered here, will be applied with $h=1$.

As already mentioned there are several ways to construct discrete Dirac operators; see [19, 12, 16, 11. For our purpose we will use the so-called Hermitian setting 
to construct a Dirac operator factorizing the star Laplacian. To this end we split each basis element $\mathbf{e}_{k}$ into two basis elements $\mathbf{e}_{k}^{+}$and $\mathbf{e}_{k}^{-}$, cf. [11, and we consider the free algebra over $\left\{\mathbf{e}_{k}^{+}, \mathbf{e}_{k}^{-}\right\}$, satisfying the following relations:

$$
\begin{aligned}
\mathbf{e}_{j}^{-} \mathbf{e}_{k}^{-}+\mathbf{e}_{k}^{-} \mathbf{e}_{j}^{-} & =0, \\
\mathbf{e}_{j}^{+} \mathbf{e}_{k}^{+}+\mathbf{e}_{k}^{+} \mathbf{e}_{j}^{+} & =0, \\
\mathbf{e}_{j}^{+} \mathbf{e}_{k}^{-}+\mathbf{e}_{k}^{-} \mathbf{e}_{j}^{+} & =\delta_{j k} .
\end{aligned}
$$

With this basis we construct a discrete Dirac operator as

$$
D=\sum_{j=1}^{m}\left(\mathbf{e}_{j}^{+} \Delta_{j}^{+}+\mathbf{e}_{j}^{-} \Delta_{j}^{-}\right)
$$

for which it is directly seen that $D^{2}=\Delta$, the star Laplacian.

\section{S-WEyl RElations AND VeCtor VARIABle CALCUlus}

The vector variable corresponding to the Dirac operator (1) is given by

$$
X=\sum_{j=1}^{m}\left(\mathbf{e}_{j}^{+} X_{j}^{-}+\mathbf{e}_{j}^{-} X_{j}^{+}\right) .
$$

Observe that, while the differences $\Delta_{j}^{+}$and $\Delta_{j}^{-}$mutually commute, and they also commute with $X_{k}^{ \pm}(j \neq k)$, the same will no longer be true for their interaction with $X_{j}^{+}$and $X_{j}^{-}$. To overcome this problem we propose the following "skew" Weyl relations, or S-Weyl relations for short, replacing the classical ones:

$$
\begin{aligned}
\Delta_{j}^{+} X_{j}^{+}-X_{j}^{-} \Delta_{j}^{-} & =1, \\
\Delta_{j}^{-} X_{j}^{-}-X_{j}^{+} \Delta_{j}^{+} & =1 .
\end{aligned}
$$

In order to construct the appropriate Euler operator $E$, we postulate the intertwining relation

$$
D X+X D=2 E+m
$$

which holds for the Dirac operator and the vector variable in the continuous case. Thus, we need to calculate the same anticommutator $D X+X D$ in the present discrete framework. Since $\Delta_{j}^{ \pm}$commutes with $X_{k}^{ \pm}$and $\mathbf{e}_{j}^{+}$anticommutes with $\mathbf{e}_{k}^{-}$ $(j \neq k)$, we only have to consider the part where $j=k$. Hence, we get

$$
D X+X D=\sum_{j=1}^{m}\left(\mathbf{e}_{j}^{+} \mathbf{e}_{j}^{-} \Delta_{j}^{+} X_{j}^{+}+\mathbf{e}_{j}^{-} \mathbf{e}_{j}^{+} \Delta_{j}^{-} X_{j}^{-}+\mathbf{e}_{j}^{+} \mathbf{e}_{j}^{-} X_{j}^{-} \Delta_{j}^{-}+\mathbf{e}_{j}^{-} \mathbf{e}_{j}^{+} X_{j}^{+} \Delta_{j}^{+}\right)
$$

while all terms with either $\left(\mathbf{e}_{j}^{+}\right)^{2}$ or $\left(\mathbf{e}_{j}^{-}\right)^{2}$ are vanishing. Now, using the S-Weyl relations (2)-(3) we obtain

$$
\begin{aligned}
D X+X D & =\sum_{j=1}^{m}\left[\mathbf{e}_{j}^{+} \mathbf{e}_{j}^{-}\left(1+2 X_{j}^{-} \Delta_{j}^{-}\right)+\mathbf{e}_{j}^{-} \mathbf{e}_{j}^{+}\left(1+2 X_{j}^{+} \Delta_{j}^{+}\right)\right] \\
& =\sum_{j=1}^{m}\left(\mathbf{e}_{j}^{+} \mathbf{e}_{j}^{-}+\mathbf{e}_{j}^{-} \mathbf{e}_{j}^{+}\right)+2 \sum_{j=1}^{m}\left(\mathbf{e}_{j}^{+} \mathbf{e}_{j}^{-} X_{j}^{-} \Delta_{j}^{-}+\mathbf{e}_{j}^{-} \mathbf{e}_{j}^{+} X_{j}^{+} \Delta_{j}^{+}\right) \\
& =m+2 \sum_{j=1}^{m}\left(\mathbf{e}_{j}^{+} \mathbf{e}_{j}^{-} X_{j}^{-} \Delta_{j}^{-}+\mathbf{e}_{j}^{-} \mathbf{e}_{j}^{+} X_{j}^{+} \Delta_{j}^{+}\right),
\end{aligned}
$$


which suggests defining the Euler operator as

$$
E=\sum_{j=1}^{m}\left(\mathbf{e}_{j}^{+} \mathbf{e}_{j}^{-} X_{j}^{-} \Delta_{j}^{-}+\mathbf{e}_{j}^{-} \mathbf{e}_{j}^{+} X_{j}^{+} \Delta_{j}^{+}\right) .
$$

Note that, although unlike the classical case, the Euler operator is no longer a scalar operator, it will reduce to a scalar one in the case of spinor-valued functions. Furthermore, it is well defined, as we may conclude by considering the commutator $[D, E]$. Similarly as above we only have to consider the terms where $k=j$, which yields

$$
\begin{aligned}
D E-E D= & \sum_{j=1}^{m}\left[\left(\mathbf{e}_{j}^{+} \Delta_{j}^{+}\right)\left(\mathbf{e}_{j}^{+} \mathbf{e}_{j}^{-} X_{j}^{-} \Delta_{j}^{-}\right)+\left(\mathbf{e}_{j}^{+} \Delta_{j}^{+}\right)\left(\mathbf{e}_{j}^{-} \mathbf{e}_{j}^{+} X_{j}^{+} \Delta_{j}^{+}\right)\right. \\
& \left.+\left(\mathbf{e}_{j}^{-} \Delta_{j}^{-}\right)\left(\mathbf{e}_{j}^{+} \mathbf{e}_{j}^{-} X_{j}^{-} \Delta_{j}^{-}\right)+\left(\mathbf{e}_{j}^{-} \Delta_{j}^{-}\right)\left(\mathbf{e}_{j}^{-} \mathbf{e}_{j}^{+} X_{j}^{+} \Delta_{j}^{+}\right)\right] \\
& -\sum_{j=1}^{m}\left[\left(\mathbf{e}_{j}^{+} \mathbf{e}_{j}^{-} X_{j}^{-} \Delta_{j}^{-}\right)\left(\mathbf{e}_{j}^{+} \Delta_{j}^{+}\right)+\left(\mathbf{e}_{j}^{-} \mathbf{e}_{j}^{+} X_{j}^{+} \Delta_{j}^{+}\right)\left(\mathbf{e}_{j}^{+} \Delta_{j}^{+}\right)\right. \\
& \left.+\left(\mathbf{e}_{j}^{+} \mathbf{e}_{j}^{-} X_{j}^{-} \Delta_{j}^{-}\right)\left(\mathbf{e}_{j}^{-} \Delta_{j}^{-}\right)+\left(\mathbf{e}_{j}^{-} \mathbf{e}_{j}^{+} X_{j}^{+} \Delta_{j}^{+}\right)\left(\mathbf{e}_{j}^{-} \Delta_{j}^{-}\right)\right] .
\end{aligned}
$$

Again invoking $\left(\mathbf{e}_{j}^{+}\right)^{2}=\left(\mathbf{e}_{j}^{-}\right)^{2}=0$ we obtain

$$
\begin{aligned}
D E-E D= & \sum_{j=1}^{m}\left[\left(\mathbf{e}_{j}^{+} \Delta_{j}^{+}\right)\left(\mathbf{e}_{j}^{-} \mathbf{e}_{j}^{+} X_{j}^{+} \Delta_{j}^{+}\right)+\left(\mathbf{e}_{j}^{-} \Delta_{j}^{-}\right)\left(\mathbf{e}_{j}^{+} \mathbf{e}_{j}^{-} X_{j}^{-} \Delta_{j}^{-}\right)\right] \\
& -\sum_{j=1}^{m}\left[\left(\mathbf{e}_{j}^{+} \mathbf{e}_{j}^{-} X_{j}^{-} \Delta_{j}^{-}\right)\left(\mathbf{e}_{j}^{+} \Delta_{j}^{+}\right)+\left(\mathbf{e}_{j}^{-} \mathbf{e}_{j}^{+} X_{j}^{+} \Delta_{j}^{+}\right)\left(\mathbf{e}_{j}^{-} \Delta_{j}^{-}\right)\right] \\
= & \sum_{j=1}^{m}\left(\mathbf{e}_{j}^{+} \Delta_{j}^{+} X_{j}^{+} \Delta_{j}^{+}+\mathbf{e}_{j}^{-} \Delta_{j}^{-} X_{j}^{-} \Delta_{j}^{-}\right) \\
& -\sum_{j=1}^{m}\left(\mathbf{e}_{j}^{+} X_{j}^{-} \Delta_{j}^{-} \Delta_{j}^{+}+\mathbf{e}_{j}^{-} X_{j}^{+} \Delta_{j}^{+} \Delta_{j}^{-}\right) \\
= & \sum_{j=1}^{m}\left[\mathbf{e}_{j}^{+}\left(X_{j}^{-} \Delta_{j}^{-} \Delta_{j}^{+}+\Delta_{j}^{+}\right)+\mathbf{e}_{j}^{-}\left(X_{j}^{+} \Delta_{j}^{+} \Delta_{j}^{-}+\Delta_{j}^{-}\right)\right] \\
& -\sum_{j=1}^{m}\left(\mathbf{e}_{j}^{+} X_{j}^{-} \Delta_{j}^{-} \Delta_{j}^{+}+\mathbf{e}_{j}^{-} X_{j}^{+} \Delta_{j}^{+} \Delta_{j}^{-}\right) \\
= & \sum_{j=1}^{m}\left(\mathbf{e}_{j}^{+} \Delta_{j}^{+}+\mathbf{e}_{j}^{-} \Delta_{j}^{-}\right)=D .
\end{aligned}
$$

In other words, the Euler operator $E$ (5) still satisfies the intertwining relation with the Dirac operator

$$
D E=E D+D
$$

which holds in the continuous setting. In the same way we can show the corresponding formula for the intertwining of the vector variable $X$ and the Euler operator $E$, 
viz.

$$
E X=X E+X
$$

Remark 3.1. Note that (6) also allows us to introduce the Gamma operator, which is given in the continuous case by $[X, D]=2 \Gamma-m$; see [1]. Thus, in the present framework, we define

$$
\Gamma=\frac{X D-D X+m}{2}
$$

whence we obtain, on the level of coordinate operators, that

$$
\Gamma=\sum_{j<k}\left[\mathbf{e}_{j}^{+} \mathbf{e}_{k}^{-}\left(X_{k}^{-} \Delta_{j}^{-}-X_{j}^{-} \Delta_{k}^{-}\right)+\mathbf{e}_{j}^{-} \mathbf{e}_{k}^{+}\left(X_{k}^{+} \Delta_{j}^{+}-X_{j}^{+} \Delta_{k}^{+}\right)\right] .
$$

Now it is easy to check that, as in the continuous case, $[E, \Gamma]=0$. Indeed, since $X D=E+\Gamma$ and $X D E=X(E D+D)=E X D-X D+X D=E X D$, the desired relation follows.

The above relations now allow us to define discrete homogeneity of a polynomial by means of the Euler operator.

Definition 3.1. A discrete polynomial $P$ is called homogeneous of degree $k$ if and only if $E P=k P$.

In combination with (6), this definition also implies that $X$ applied to a discrete homogeneous polynomial will result in a discrete homogeneous polynomial of degree $k+1$, and thus may be seen as a raising operator. Moreover, the S-Weyl relations (2)-(3) will now enable us to construct homogeneous polynomials recursively. Indeed, we have

$$
\begin{aligned}
\Delta_{j}^{+} X_{j}^{+}\left[P_{k}\right]-X_{j}^{-} \Delta_{j}^{-}\left[P_{k}\right] & =P_{k}, \\
\Delta_{j}^{-} X_{j}^{-}\left[P_{k}\right]-X_{j}^{+} \Delta_{j}^{+}\left[P_{k}\right] & =P_{k},
\end{aligned}
$$

showing that, if $P_{k}$ is given, via a simple polynomial ansatz one can determine $X_{j}^{ \pm}\left[P_{k}\right]$, and thus also $X\left[P_{k}\right]$ from $P_{k}$.

As a first step towards this desired construction of homogeneous polynomials, we will start by calculating the action of $X_{j}^{ \pm}$on the classical homogeneous powers $x_{j}^{k}$. Denoting $\left(P_{k+1}^{j}\right)^{ \pm} \equiv X_{j}^{ \pm}\left[x_{j}^{k}\right]$ we get

$$
\begin{aligned}
\Delta_{j}^{+}\left[\left(P_{k+1}^{j}\right)^{+}\right] & =x_{j}^{k}+X_{j}^{-} \Delta_{j}^{-}\left[x_{j}^{k}\right], \\
\Delta_{j}^{-}\left[\left(P_{k+1}^{j}\right)^{-}\right] & =x_{j}^{k}+X_{j}^{+} \Delta_{j}^{+}\left[x_{j}^{k}\right] .
\end{aligned}
$$

Now, denoting by $c_{l}^{k}$ the $l$ th coefficient in $X_{j}^{-}\left[x_{j}^{k}\right]$ and by $d_{l}^{k}$ the $l$ th coefficient in $X_{j}^{+}\left[x_{j}^{k}\right]$, i.e. putting

$$
\begin{aligned}
& \left(P_{k+1}^{j}\right)^{+}=d_{k+1}^{k} x_{j}^{k+1}+d_{k}^{k} x_{j}^{k}+\ldots+d_{1}^{k} x_{j}, \\
& \left(P_{k+1}^{j}\right)^{-}=c_{k+1}^{k} x_{j}^{k+1}+c_{k}^{k} x_{j}^{k}+\ldots+c_{1}^{k} x_{j},
\end{aligned}
$$


we are led to the following linear system for the polynomial coefficients of $\left(P_{k+1}^{j}\right)^{-}$:

$$
\begin{gathered}
\left(\begin{array}{ccccc}
\left(\begin{array}{c}
k+1 \\
1
\end{array}\right) & 0 & 0 & \ldots & 0 \\
-\left(\begin{array}{c}
k+1 \\
2
\end{array}\right) & \left(\begin{array}{c}
k \\
1
\end{array}\right) & 0 & \ldots & 0 \\
\left(\begin{array}{c}
k+1 \\
3
\end{array}\right) & -\left(\begin{array}{c}
k \\
2
\end{array}\right) & \left(\begin{array}{c}
k-1 \\
1
\end{array}\right) & & 0 \\
\vdots & \vdots & \vdots & \ddots & \vdots \\
(-1)^{k}\left(\begin{array}{c}
k+1 \\
k+1
\end{array}\right) & (-1)^{k-1}\left(\begin{array}{c}
k \\
k
\end{array}\right) & (-1)^{k}\left(\begin{array}{c}
k-1 \\
k-1
\end{array}\right) & \ldots & \left(\begin{array}{l}
1 \\
1
\end{array}\right)
\end{array}\right)\left(\begin{array}{c}
c_{k+1}^{k} \\
c_{k}^{k} \\
c_{k-1}^{k} \\
\vdots \\
c_{1}^{k}
\end{array}\right) \\
=\left(\begin{array}{ccccc}
d_{k}^{k-1} & 0 & 0 & \ldots & 0 \\
d_{k-1}^{k-1} & d_{k-1}^{k-2} & 0 & \ldots & 0 \\
\vdots & \vdots & \vdots & \ddots & \vdots \\
d_{1}^{k-1} & d_{1}^{k-2} & \ldots & d_{1}^{1} & 0 \\
0 & 0 & \ldots & 0 & 0
\end{array}\right)\left(\begin{array}{c}
\left(\begin{array}{c}
k \\
1
\end{array}\right) \\
\left(\begin{array}{c}
k \\
2
\end{array}\right) \\
\vdots \\
\left(\begin{array}{c}
k \\
k
\end{array}\right) \\
0
\end{array}\right)+\left(\begin{array}{c}
1 \\
0 \\
\vdots \\
0 \\
0
\end{array}\right)
\end{gathered}
$$

while a similar system holds for the coefficients of $\left(P_{k+1}^{j}\right)^{+}$:

$$
\begin{aligned}
& \left(\begin{array}{ccccc}
\left(\begin{array}{c}
k+1 \\
1
\end{array}\right) & 0 & 0 & \ldots & 0 \\
\left(\begin{array}{c}
k+1 \\
2
\end{array}\right) & \left(\begin{array}{c}
k \\
1
\end{array}\right) & 0 & \ldots & 0 \\
\left(\begin{array}{c}
k+1 \\
3
\end{array}\right) & \left(\begin{array}{c}
k \\
2
\end{array}\right) & \left(\begin{array}{c}
k-1 \\
1
\end{array}\right) & & 0 \\
\vdots & \vdots & \vdots & \ddots & \vdots \\
\left(\begin{array}{c}
k+1 \\
k+1
\end{array}\right) & \left(\begin{array}{c}
k \\
k
\end{array}\right) & \left(\begin{array}{c}
k-1 \\
k-1
\end{array}\right) & \ldots & \left(\begin{array}{l}
1 \\
1
\end{array}\right)
\end{array}\right)\left(\begin{array}{c}
d_{k+1}^{k} \\
d_{k}^{k} \\
d_{k-1}^{k} \\
\vdots \\
d_{1}^{k}
\end{array}\right) \\
& =\left(\begin{array}{ccccc}
c_{k}^{k-1} & 0 & 0 & \ldots & 0 \\
c_{k-1}^{k-1} & c_{k-1}^{k-2} & 0 & \ldots & 0 \\
\vdots & \vdots & \vdots & \ddots & \vdots \\
c_{1}^{k-1} & c_{1}^{k-2} & \ldots & c_{1}^{1} & 0 \\
0 & 0 & \ldots & 0 & 0
\end{array}\right)\left(\begin{array}{c}
\left(\begin{array}{c}
k \\
1
\end{array}\right) \\
-\left(\begin{array}{c}
k \\
2
\end{array}\right) \\
\vdots \\
(-1)^{k}\left(\begin{array}{c}
k \\
k
\end{array}\right) \\
0
\end{array}\right)+\left(\begin{array}{c}
1 \\
0 \\
\vdots \\
0 \\
0
\end{array}\right) .
\end{aligned}
$$

From these systems the following results are obtained for low degrees:

$$
\begin{aligned}
\left(P_{1}^{j}\right)^{+} \equiv X_{j}^{+}[1] & =x_{j}, \\
\left(P_{1}^{j}\right)^{-} \equiv X_{j}^{-}[1] & =x_{j}, \\
\left(P_{2}^{j}\right)^{+} \equiv X_{j}^{+}\left[x_{j}\right] & =x_{j}^{2}-x_{j}, \\
\left(P_{2}^{j}\right)^{-} \equiv X_{j}^{-}\left[x_{j}\right] & =x_{j}^{2}+x_{j}, \\
\left(P_{3}^{j}\right)^{+} \equiv X_{j}^{+}\left[x_{j}^{2}\right] & =x_{j}^{3}-x_{j}^{2}, \\
\left(P_{3}^{j}\right)^{-} \equiv X_{j}^{-}\left[x_{j}^{2}\right] & =x_{j}^{3}+x_{j}^{2} .
\end{aligned}
$$


While, as expected, the action of our vector variable is not as simple as in the standard Weyl case where we would have a Rodrigues' formula for $X_{j}^{+}$and $X_{j}^{-}$ independently, our polynomials are still of the form

$$
X_{j}^{ \pm}\left[x_{j}^{k}\right]=x_{j}^{k+1}+\text { lower order terms. }
$$

In particular, the coefficients of the first 11 polynomials are given in the matrices below, where the $i$ th column contains the coefficients of $X_{j}^{ \pm}\left[x_{j}^{11-i}\right]$, while the $j$ th row provides the respective coefficients of $\left(x_{j}\right)^{12-i}$, the last row indeed corresponding to $x_{j}$ since the constant term always is zero. For the action of $X_{j}^{+}$this yields

$$
M^{+}=\left[\begin{array}{rrrrrrrrrrr}
1 & 0 & 0 & 0 & 0 & 0 & 0 & 0 & 0 & 0 & 0 \\
-5 & 1 & 0 & 0 & 0 & 0 & 0 & 0 & 0 & 0 & 0 \\
0 & -5 & 1 & 0 & 0 & 0 & 0 & 0 & 0 & 0 & 0 \\
30 & 0 & -4 & 1 & 0 & 0 & 0 & 0 & 0 & 0 & 0 \\
0 & 30 & 0 & -4 & 1 & 0 & 0 & 0 & 0 & 0 & 0 \\
-126 & 0 & 14 & 0 & -3 & 1 & 0 & 0 & 0 & 0 & 0 \\
0 & -126 & 0 & 14 & 0 & -3 & 1 & 0 & 0 & 0 & 0 \\
255 & 0 & -28 & 0 & 5 & 0 & -2 & 1 & 0 & 0 & 0 \\
0 & 255 & 0 & -28 & 0 & 5 & 0 & -2 & 1 & 0 & 0 \\
-155 & 0 & 17 & 0 & -3 & 0 & 1 & 0 & -1 & 1 & 0 \\
0 & -155 & 0 & 17 & 0 & -3 & 0 & 1 & 0 & -1 & 1
\end{array}\right]
$$

while for the action of $X_{j}^{-}$we have

$$
M^{-}=\left[\begin{array}{rrrrrrrrrrr}
1 & 0 & 0 & 0 & 0 & 0 & 0 & 0 & 0 & 0 & 0 \\
5 & 1 & 0 & 0 & 0 & 0 & 0 & 0 & 0 & 0 & 0 \\
0 & 5 & 1 & 0 & 0 & 0 & 0 & 0 & 0 & 0 & 0 \\
-30 & 0 & 4 & 1 & 0 & 0 & 0 & 0 & 0 & 0 & 0 \\
0 & -30 & 0 & 4 & 1 & 0 & 0 & 0 & 0 & 0 & 0 \\
126 & 0 & -14 & 0 & 3 & 1 & 0 & 0 & 0 & 0 & 0 \\
0 & 126 & 0 & -14 & 0 & 3 & 1 & 0 & 0 & 0 & 0 \\
-255 & 0 & 28 & 0 & -5 & 0 & 2 & 1 & 0 & 0 & 0 \\
0 & -255 & 0 & 28 & 0 & -5 & 0 & 2 & 1 & 0 & 0 \\
155 & 0 & -17 & 0 & 3 & 0 & -1 & 0 & 1 & 1 & 0 \\
0 & 155 & 0 & -17 & 0 & 3 & 0 & -1 & 0 & 1 & 1
\end{array}\right] .
$$

These lists of coefficients now allow for two direct observations. First, as could be expected, the coefficients of $X_{j}^{+}$and those of $X_{j}^{-}$only differ in a change of signs of all but the leading terms, and secondly, the transition from an even to an odd degree polynomial just consists in a forward shift of the coefficients. However, there is more.

In the structure of the above matrix entries one may recognize the Euler polynomials of even degree. Indeed, these polynomials, which we will denote by $E_{n}(x)$, 
show the following property:

$$
E_{n}(x)-(-1)^{n+1} E_{n}(-x)=2 x^{n},
$$

which, for $n$ even, exactly corresponds to the observed similarity between the columns of $M^{+}$and $M^{-}$. In order to formulate and verify our observation, we will need an auxiliary result, as formulated in the following lemma.

Lemma 3.1. Let $E_{n}(x)$ be the Euler polynomial of degree $n, n \in \mathbb{N}$. Denoting by $e_{\ell}^{n}$ the $\ell$ th coefficient in $E_{n}(x)$, i.e. putting

$$
E_{n}(x)=\sum_{\ell=0}^{n} e_{\ell}^{n} x^{\ell}
$$

the following relation holds for $\ell=0, \ldots, n-1$ :

$$
\sum_{j=\ell}^{n}\left(\begin{array}{c}
n \\
j
\end{array}\right) e_{\ell}^{j}=-e_{\ell}^{n}
$$

while $e_{n}^{n}=1$.

Proof. Combine the well-known recurrence relations $E_{n}(x+1)=2 x^{n}-E_{n}(x)$ and

$$
E_{n}(x+1)=\sum_{j=0}^{n}\left(\begin{array}{l}
n \\
j
\end{array}\right) E_{j}(x) .
$$

The above observation is then formulated in the following theorem.

Theorem 3.1. The polynomials $\left(P_{k+1}^{j}\right)^{ \pm}$, resulting from the action of $X_{j}^{ \pm}$on the classical homogeneous powers $x_{j}^{k}, k \in \mathbb{N}$, can be written in terms of the Euler polynomials of even degree. More precisely, for $k$ odd, one has

$$
\begin{aligned}
& \left(P_{k+1}^{j}\right)^{+}=X_{j}^{+}\left(x_{j}^{k}\right)=E_{k+1}\left(x_{j}\right), \\
& \left(P_{k+1}^{j}\right)^{-}=X_{j}^{-}\left(x_{j}^{k}\right)=E_{k+1}\left(-x_{j}\right),
\end{aligned}
$$

while for $k$ even, one has

$$
\begin{aligned}
& \left(P_{k+1}^{j}\right)^{+}=X_{j}^{+}\left(x_{j}^{k}\right)=x E_{k}\left(x_{j}\right), \\
& \left(P_{k+1}^{j}\right)^{-}=X_{j}^{-}\left(x_{j}^{k}\right)=x E_{k}\left(-x_{j}\right) .
\end{aligned}
$$

Proof. The proof of (10) is given below by induction; (11)-(13) can be proven along similar lines. So take $k$ odd, and denote for simplicity $X_{j}^{+}\left(x_{j}^{k}\right)$ as $X^{+}\left(x^{k}\right)$. Since $X^{+}\left(x^{k}\right)$ is completely determined by the S-Weyl relation (2), it suffices to prove that

$$
\Delta^{+} E_{k+1}(x)-X^{-} \Delta^{-}\left(x^{k}\right)=x^{k} .
$$

Using (9) we directly obtain

$$
\Delta^{+} E_{k+1}(x)-X^{-} \Delta^{-}\left(x^{k}\right)=\sum_{r=0}^{k}\left(\begin{array}{c}
k+1 \\
r
\end{array}\right) E_{r}(x)+\sum_{\ell=0}^{k-1}(-1)^{k-\ell}\left(\begin{array}{c}
k \\
\ell
\end{array}\right) X^{-}\left(x^{\ell}\right) .
$$


Since $\ell<k$, we may now apply the induction hypothesis, which yields

$$
\begin{aligned}
\Delta^{+} E_{k+1}(x)-X^{-} \Delta^{-}\left(x^{k}\right) & \\
= & \sum_{r=0}^{k}\left(\begin{array}{c}
k+1 \\
r
\end{array}\right) E_{r}(x)-\sum_{\substack{\ell=0 \\
\text { ODD }}}^{k-1}\left(\begin{array}{l}
k \\
\ell
\end{array}\right) E_{\ell+1}(x)+\sum_{\substack{\ell=0 \\
\text { EVEN }}}^{k-1}\left(\begin{array}{l}
k \\
\ell
\end{array}\right) x E_{\ell}(x) \\
& +2 \sum_{\ell=0}^{k-1}(-1)^{k-\ell}\left(\begin{array}{l}
k \\
\ell
\end{array}\right) x^{\ell+1},
\end{aligned}
$$

where we have also invoked (7). Rewriting the Euler polynomials explicitly as in (8), we arrive at

$$
\begin{aligned}
\Delta^{+} E_{k+1}(x)-X^{-} \Delta^{-}\left(x^{k}\right) & \\
= & \sum_{r=0}^{k}\left(\begin{array}{c}
k+1 \\
r
\end{array}\right)\left(x^{r}+\sum_{\ell=0}^{r-1} e_{\ell}^{r} x^{\ell}\right)-\sum_{\substack{\ell=0 \\
\text { ODD }}}^{k-1}\left(\begin{array}{c}
k \\
\ell
\end{array}\right)\left(x^{\ell+1}+\sum_{\substack{p=0 \\
\text { ODD }}}^{\ell} e_{p}^{\ell+1} x^{p}\right) \\
& +\sum_{\substack{\ell=0 \\
\text { EVEN }}}^{k-1}\left(\begin{array}{c}
k \\
\ell
\end{array}\right)\left(x^{\ell+1}+\sum_{\substack{p=0 \\
\text { ODD }}}^{\ell-1} e_{p}^{\ell} x^{p+1}\right)+2 \sum_{\ell=0}^{k-1}(-1)^{k-\ell}\left(\begin{array}{c}
k \\
\ell
\end{array}\right) x^{\ell+1} .
\end{aligned}
$$

Since $k$ is odd, the coefficient of $x^{k}$ in the previous expression equals $\left(\begin{array}{c}k+1 \\ k\end{array}\right)-\left(\begin{array}{c}k \\ k-1\end{array}\right)=$ 1. It thus remains to prove that the coefficients of all lower order terms are zero. For $s$ odd, the coefficient of $x^{s}$ is

$$
\begin{aligned}
& \left(\begin{array}{c}
k+1 \\
s
\end{array}\right)+\sum_{\ell=s+1}^{k}\left(\begin{array}{c}
k+1 \\
\ell
\end{array}\right) e_{s}^{\ell}-\sum_{\substack{\ell=s \\
\text { ODD }}}^{k-1}\left(\begin{array}{l}
k \\
\ell
\end{array}\right) e_{s}^{\ell+1}+\left(\begin{array}{c}
k \\
s-1
\end{array}\right)-2\left(\begin{array}{c}
k \\
s-1
\end{array}\right) \\
& =\left(\begin{array}{c}
k \\
s
\end{array}\right)+\sum_{\substack{\ell=s+1 \\
\operatorname{EVEN}}}^{k}\left(\begin{array}{l}
k \\
\ell
\end{array}\right) e_{s}^{\ell},
\end{aligned}
$$

where we have used the fact that $e_{s}^{\ell}=0$ whenever $s$ and $\ell$ both are odd. From Lemma 3.1 it then follows that

$$
\sum_{\ell=s+1}^{k}\left(\begin{array}{l}
k \\
\ell
\end{array}\right) e_{s}^{\ell}=-e_{s}^{k}-\left(\begin{array}{l}
k \\
s
\end{array}\right)=-\left(\begin{array}{l}
k \\
s
\end{array}\right)
$$

since also $e_{s}^{k}=0$ as both $k$ and $s$ are odd. Thus we have obtained that the coefficient of $x^{s}$ indeed equals zero, for $s$ odd. Proceeding similarly, one proves the same for $s$ even.

\section{FischeR DECOMPOSITION}

One of the basic tools in constructing a discrete function theory is the Fischer decomposition for homogeneous polynomials. The traditional Fischer decomposition in harmonic analysis yields an orthogonal decomposition of the space $\mathcal{P}_{k}$ of homogeneous polynomials on $\mathbb{R}^{m}$ of given homogeneity $k$ in terms of spaces of harmonic homogeneous polynomials. In classical continuous Clifford analysis, a refinement 
was obtained, yielding an orthogonal decomposition with respect to the so-called Fischer inner product of homogeneous polynomials, given by

$$
\langle P(\underline{x}), Q(\underline{x})\rangle=\operatorname{Sc}\left[\overline{P\left(\partial_{\underline{x}}\right)} Q(\underline{x})\right]
$$

in terms of spaces of monogenic polynomials, i.e. null solutions of the considered Dirac operator; see e.g. 6]. Here, the notation Sc[.] stands for taking the scalar part of a Clifford algebra-valued expression, while $\overline{P\left(\partial_{x}\right)}$ is a differential operator obtained by replacing in the polynomial $P$ each variable $x_{j}$ by the corresponding partial derivative $\partial_{x_{j}}$ and applying Clifford conjugation. This Fischer inner product results from a duality argument, called Fischer duality, between the algebra of vector variables and the algebra of operators. Generalizations as well as refinements of the Fischer decomposition in other Clifford analysis frameworks can be found e.g. in [5, 8, 10, 17.

Formally, in the present discrete context, a Fischer inner product of two homogeneous polynomials $P$ and $Q$ could be expected to be of the following form:

$$
\operatorname{Sc}\left[P\left(\Delta_{j}^{ \pm}\right)^{\dagger} Q\left(X_{j}^{ \pm}\right)\right][1]
$$

where ${ }^{\dagger}$ now denotes the Hermitian conjugation, accounting for the chosen Hermitian framework, and sending the basis vector $e_{j}^{+}$into $e_{j}^{-}$(and vice versa). However, a fundamental problem arises. Since $\Delta_{j}^{+}$commutes with $\Delta_{j}^{-}$, but $X_{j}^{+}$does not commute with $X_{j}^{-}$, a direct duality argument sending $X_{j}^{ \pm}$into $\Delta_{j}^{ \pm}$is no longer available, whence we need to define the Fischer inner product in another way.

To overcome this problem we propose to work directly on the level of the coordinate variable

$$
\xi_{j}=X_{j}^{+} e_{j}^{-}+X_{j}^{-} e_{j}^{+},
$$

enabling us to work simultaneously on the considered graph and its dual. Similarly, we also consider the coordinate difference operator

$$
\delta_{j}=\mathbf{e}_{j}^{+} \Delta_{j}^{+}+\mathbf{e}_{j}^{-} \Delta_{j}^{-} .
$$

In this way, we have in fact decomposed the discrete Dirac operator and the vector variable respectively as

$$
D=\sum_{j=1}^{m} \delta_{j}, \quad X=\sum_{j=1}^{m} \xi_{j} .
$$

On account of the skew Weyl relations (2)-(3) for $X_{j}^{ \pm}$and $\Delta_{j}^{ \pm}$, it is easily seen that $\xi_{j}$ and $\delta_{j}$ satisfy the Weyl relations

$$
\begin{aligned}
\delta_{j} \xi_{j}-\xi_{j} \delta_{j} & =1, \\
\delta_{k} \xi_{j}+\xi_{j} \delta_{k} & =0, \quad k \neq j .
\end{aligned}
$$

Moreover, using the intertwining relation $E X=X(E+1)$, it directly follows that

$$
E \xi_{j}=\xi_{j}(E+1) .
$$

Observe that, while

$$
\xi_{j}^{2}=e_{j}^{+} e_{j}^{-} X_{j}^{-} X_{j}^{+}+e_{j}^{-} e_{j}^{+} X_{j}^{+} X_{j}^{-}
$$

is not a scalar, it does form a one-dimensional subspace. Moreover, while the $\delta_{j}$ 's are not scalar operators one still gets

$$
\xi_{j}\left[x_{j}^{k}\right]=\left(e_{j}^{+}+e_{j}^{-}\right) x_{j}^{k+1}+\left(e_{j}^{+}-e_{j}^{-}\right) x_{j}^{k}+\text { lower order terms. }
$$


In particular, we get for the action of natural powers of $\xi_{j}$ on the ground state 1 ,

$$
\begin{aligned}
\xi_{j}^{2 k}[1] & =x_{j}^{2 k}+c\left(e_{j}^{+} e_{j}^{-}-e_{j}^{-} e_{j}^{+}\right) x_{j}^{2 k-1}+\text { lower order terms, } \\
\xi_{j}^{2 k+1}[1] & =\left(e_{j}^{+}+e_{j}^{-}\right) x_{j}^{2 k+1}+\text { lower order terms, }
\end{aligned}
$$

in which $c$ denotes a constant and the absolute term (corresponding to $x_{j}^{0}$ ) always is zero. For the sake of completeness, we mention some explicit results for low degrees:

$$
\begin{aligned}
\xi_{j}[1] & =\left(e_{j}^{+}+e_{j}^{-}\right) x_{j}, \\
\xi_{j}^{2}[1] & =x_{j}^{2}+\left(e_{j}^{+} e_{j}^{-}-e_{j}^{-} e_{j}^{+}\right) x_{j}, \\
\xi_{j}^{3}[1] & =\left(e_{j}^{+}+e_{j}^{-}\right) x_{j}^{3}-\left(e_{j}^{+}+e_{j}^{-}\right) x_{j}, \\
\xi_{j}^{4}[1] & =x_{j}^{4}+2\left(e_{j}^{+} e_{j}^{-}-e_{j}^{-} e_{j}^{+}\right) x_{j}^{3}-x_{j}^{2}-2\left(e_{j}^{+} e_{j}^{-}-e_{j}^{-} e_{j}^{+}\right) x_{j}, \\
\xi_{j}^{5}[1] & =\left(e_{j}^{+}+e_{j}^{-}\right) x_{j}^{5}-5\left(e_{j}^{+}+e_{j}^{-}\right) x_{j}^{3}+4\left(e_{j}^{+}+e_{j}^{-}\right) x_{j} .
\end{aligned}
$$

By means of the above coordinate operators, we may now introduce in a correct way the Fischer inner product of homogeneous polynomials in the present context.

Definition 4.1. The Fischer inner product of two polynomials $P$ and $Q$, being homogeneous of the respective degrees $k$ and $m$, is given by

$$
\langle P, Q\rangle=\mathrm{Sc}\left[P(\delta)^{\dagger} Q(\xi)[1](0)\right],
$$

where $Q(\xi)$ denotes the operator obtained by substituting in the polynomial $Q$ the variable $x_{j}$ by $\xi_{j}$, and $P(\delta)$ denotes the difference operator obtained by substituting in the polynomial $P$ the variable $x_{j}$ by $\delta_{j}$. Both $P$ and $Q$ are then acting as operators on the ground state 1 , the result of which is evaluated at the point zero.

Clearly, the name Fischer inner product is, at this moment, not justified. To further study this Fischer inner product, and in particular, to show that it indeed represents an inner product on the space of homogeneous polynomials of given homogeneity, we first need some auxiliary results.

Lemma 4.1. For all $n \in \mathbb{N}$ we have

$$
\delta_{i}\left(\xi_{i}\right)^{n}[1]=n\left(\xi_{i}\right)^{n-1}[1] .
$$

Moreover,

$$
\left(\delta_{i}\right)^{n}\left(\xi_{i}\right)^{n}[1]=n !
$$

Proof. Observe that the Weyl relation (14) yields

$$
\delta_{i}\left(\xi_{i}\right)^{n}[1]=\left(\xi_{i}^{n-1}+\xi_{i} \delta_{i}\left(\xi_{i}\right)^{n-1}\right)[1] .
$$

The desired result then follows from a recursive application of this formula.

Lemma 4.2. For any two multi-indices $\alpha=\left(\alpha_{1}, \ldots, \alpha_{n}\right)$ and $\beta=\left(\beta_{1}, \ldots, \beta_{n}\right)$, with $|\alpha|=|\beta|$, it follows that

$$
(\delta)^{\alpha}(\xi)^{\beta}[1]=\left\{\begin{array}{lll}
\alpha ! & \text { if } & \alpha=\beta \\
0 & \text { if } & \alpha \neq \beta
\end{array}\right.
$$

where we have put $\alpha !=\alpha_{1} ! \alpha_{2} ! \ldots \alpha_{n} !$.

Proof. This follows from Lemma 4.1 . 
The above results eventually lead to the following important property.

Proposition 4.1. For polynomials $P_{k}=\sum_{|\alpha|=k}\left(\xi^{\alpha}[1]\right) p_{\alpha}$ and $Q_{k}=\sum_{|\alpha|=k}\left(\xi^{\alpha}[1]\right) q_{\alpha}$, both homogeneous of degree $k$, we obtain

$$
\left\langle P_{k}, Q_{k}\right\rangle=\sum_{|\alpha|=k} \alpha ! \mathrm{Sc}\left[p_{\alpha}^{\dagger} q_{\alpha}\right]
$$

where, again, ${ }^{\dagger}$ stands for the Hermitian conjugate.

This property obviously implies that, on the space of homogeneous polynomials of given homogeneity $k$, the Fischer inner product is positive definite; i.e. it indeed represents an inner product. Furthermore, we have the following corollary.

Corollary 4.1. For any polynomial $P_{k-1}$ of homogeneity $k-1$ and any polynomial $Q_{k}$ of homogeneity $k$, we have

$$
\left\langle X P_{k-1}, Q_{k}\right\rangle=\left\langle P_{k-1}, D Q_{k}\right\rangle .
$$

This property allows us to prove the following theorem.

Theorem 4.1. For each $k \in \mathbb{N}$ we have

$$
\Pi_{k}=\mathcal{M}_{k}+X \Pi_{k-1},
$$

where $\Pi_{k}$ denotes the space of discrete homogeneous polynomials of degree $k$ and $\mathcal{M}_{k}$ denotes the space of discrete monogenic homogeneous polynomials of degree $k$. Furthermore, the subspaces $\mathcal{M}_{k}$ and $X \Pi_{k-1}$ are orthogonal with respect to the Fischer inner product (15).

Proof. Since

$$
\Pi_{k}=X \Pi_{k-1}+\left(X \Pi_{k-1}\right)^{\perp},
$$

it suffices to prove that $\left(X \Pi_{k-1}\right)^{\perp}=\mathcal{M}_{k-1}$. To this end, assume that, for some $P_{k} \in \Pi_{k}$ we have

$$
\left\langle X P_{k-1}, P_{k}\right\rangle=0, \quad \text { for all } P_{k-1} \in \Pi_{k-1} .
$$

On account of Corollary 4.1 we then have that

$$
\left\langle P_{k-1}, D P_{k}\right\rangle=0, \quad \text { for all } P_{k-1} \in \Pi_{k-1} \text {. }
$$

As $D P_{k} \in \Pi_{k-1}$ we obtain that $D P_{k}=0$, or that $P_{k} \in \mathcal{M}_{k}$. This means that $\left(X \Pi_{k-1}\right)^{\perp} \subset \mathcal{M}_{k-1}$. Conversely, take $P_{k} \in \mathcal{M}_{k}$. Then we have, for any $P_{k-1} \in$ $\Pi_{k-1}$, that

$$
\left\langle X P_{k-1}, P_{k}\right\rangle=\left\langle P_{k-1}, D P_{k}\right\rangle=\left\langle P_{k-1}, 0\right\rangle=0,
$$

from which it follows that $\mathcal{M}_{k-1} \subset\left(X \Pi_{k-1}\right)^{\perp}$, and therefore $\mathcal{M}_{k-1}=\left(X \Pi_{k-1}\right)^{\perp}$.

As a result we obtain the Fischer decomposition with respect to the discrete Dirac operator $D$.

Theorem 4.2 (Fischer decomposition). Let $P_{k}$ be a discrete homogeneous polynomial of degree $k$. Then

$$
P_{k}=M_{k}+X M_{k-1}+X^{2} M_{k-2}+\ldots+X^{k} M_{0},
$$

where each $M_{j}$ denotes a homogeneous discrete monogenic polynomial of degree $j$. 
The spaces represented in the above sum still are orthogonal to each other with respect to the Fischer inner product (15). This is a consequence of the construction of the Euler operator $E$, and in particular of (44).

\section{Discrete monogenic PROJeCtion}

Finally, we want to obtain an explicit formula for the projection $\operatorname{proj}_{\mathcal{M}} P_{k}$ of a given homogeneous polynomial $P_{k}$ on the space of homogeneous discrete monogenic polynomials.

To this end we observe the following property.

Lemma 5.1. We have that, for any $n \in \mathbb{N}$,

$$
\begin{aligned}
D X^{2 n+1} & =2 n X^{2 n}+2 X^{2 n} E+m X^{2 n}-X^{2 n+1} D, \\
D X^{2 n} & =2 n X^{2 n-1}+X^{2 n} D .
\end{aligned}
$$

Proof. This follows by a direct calculation, using the intertwining relations $D X+$ $X D=2 E+m$ and $E X=X E+X$.

Applying the above lemma to a given homogeneous polynomial $P_{k}$, we obtain

$$
\begin{aligned}
D X^{2 n+1} P_{k} & =(2 n+2 k+m) X^{2 n} P_{k}-X^{2 n+1} D P_{k} \\
D X^{2 n} P_{k} & =2 n X^{2 n-1} P_{k}+X^{2 n} D P_{k}
\end{aligned}
$$

Following a well-known strategy developed in [7] for the harmonic projection of a given homogeneous polynomial, and successfully applied in the Clifford analysis context in [18], we postulate the following structure for the discrete monogenic projection $r$ of $P_{k}$ :

$$
r=P_{k}+a_{1} X D P_{k}+a_{2} X^{2} D^{2} P_{k}+\ldots+a_{k} X^{k} D^{k} P_{k},
$$

whence it only remains to express that $r \in \mathcal{M}_{k}$ by requesting that $D r=0$. This strategy then will lead to explicit expressions for the coefficients $a_{1}, \ldots, a_{k}$ in (19). For $k=2 \ell$ this yields

$$
\begin{aligned}
0= & D P_{k}+a_{1}(2(k-1)+m) D P_{k}-a_{1} X D^{2} P_{k}+2 a_{2} X D^{2} P_{k}+a_{2} X^{2} D^{3} P_{k} \\
& +a_{3}(2+2(k-3)+m) X^{2} D^{3} P_{k}-a_{3} X^{3} D^{4} P_{k}+\ldots \\
& +a_{k-1}(k+m) X^{k-2} D^{k-1} P_{k}-a_{k-1} X^{k-1} D^{k} P_{k}+a_{k} k X^{k-1} D^{k} P_{k}
\end{aligned}
$$

while for $k=2 \ell+1$ we obtain

$$
\begin{aligned}
0= & D P_{k}+a_{1}(2(k-1)+m) D P_{k}-a_{1} X D^{2} P_{k}+2 a_{2} X D^{2} P_{k}+a_{2} X^{2} D^{3} P_{k} \\
& +a_{3}(2+2(k-3)+m) X^{2} D^{3} P_{k}-a_{3} X^{3} D^{4} P_{k}+\ldots \\
& +a_{k-1}(k-1) X^{k-2} D^{k-1} P_{k}+a_{k-1} X^{k-1} D^{k} P_{k} \\
& +a_{k}(k+m-1) X^{k-1} D^{k} P_{k} .
\end{aligned}
$$

In both cases this leads to the conditions

$$
\begin{aligned}
a_{1}(2 k-2+m)+1 & =0, \\
2 a_{2}-a_{1} & =0, \\
a_{3}(2 k-4+m)+a_{2} & =0,
\end{aligned}
$$


ending with

$$
\begin{aligned}
k a_{k}-a_{k-1}= & \text { if } k=2 \ell, \\
a_{k}(k+m-1)+a_{k-1}= & 0 \quad \text { if } k=2 \ell+1 .
\end{aligned}
$$

These calculations result in the following theorem.

Theorem 5.1. The monogenic projection of a homogeneous polynomial $P_{k}$ of degree $k$, i.e. $\operatorname{proj}_{\mathcal{M}}: \Pi_{k} \mapsto \mathcal{M}_{k}$, is given by

$$
\operatorname{proj}_{\mathcal{M}} P_{k}=P_{k}+a_{1} X D P_{k}+a_{2} X^{2} D^{2} P_{k}+\ldots+a_{k} X^{k} D^{k} P_{k}
$$

with

and

$$
a_{1}=-\frac{1}{2 k-2+m}, \quad a_{2}=\frac{a_{1}}{2}, \quad a_{3}=-\frac{a_{2}}{2 k-4+m}, \ldots
$$

$$
a_{k}=\frac{a_{k-1}}{k} \text { if } k=2 \ell \quad \text { or } \quad a_{k}=-\frac{a_{k-1}}{k-1+m} \text { if } k=2 \ell+1 .
$$

Repeating a similar procedure for the respective summands in the decomposition (16), we arrive at the final result.

Theorem 5.2. Each discrete homogeneous polynomial $P_{k} \in \Pi_{k}$ can be written in a unique way as

$$
P_{k}=\sum_{j=0}^{k} X^{j} M_{k-j},
$$

where $M_{k-j} \equiv M_{k-j}\left(P_{k}\right) \in \mathcal{M}_{k-j}$ is given by

$$
M_{k-j}\left(P_{k}\right)=\frac{1}{c_{j}} \sum_{i=0}^{k-j} a_{i} X^{i} D^{i} D^{j} P_{k}
$$

where

and

$$
a_{2 i}=\frac{a_{2 i-1}}{2 i}, \quad a_{2 i+1}=-\frac{a_{2 i}}{2 k-i-1+m}
$$

$$
c_{j}= \begin{cases}(2 n) ! ! 2^{n}\left(k-2 n+\frac{m}{2}\right)_{n-1} & j=2 n, \\ (2 n) ! ! 2^{n+1}\left(k-2 n-1+\frac{m}{2}\right)_{n} & j=2 n+1 .\end{cases}
$$

Here, $(k)_{n}$ stands for the Pochhammer symbol, viz. $(k)_{n}=k(k+1) \ldots(k+n)$.

Proof. From the (unique) Fischer decomposition

$$
P_{k}=M_{k}+X M_{k-1}+\ldots+X^{k-1} M_{1}+X^{k} M_{0},
$$

we obtain that

$$
D^{j} P_{k}=D^{j} X^{j} M_{k-j}+\ldots+D^{j} X^{k-1} M_{1}+D^{j} X^{k} M_{0},
$$

whence $D^{j} X^{j} M_{k-j}$ is the discrete monogenic projection of the polynomial $D^{j} P_{k}$. Furthermore, applying (17)-(18) we obtain

$$
D^{j} X^{j} M_{k-j} j=c_{j} M_{k-j}
$$

with

$$
c_{j}= \begin{cases}(2 n) ! ! 2^{n}\left(k-2 n+\frac{m}{2}\right)_{n-1} & j=2 n, \\ (2 n) ! ! 2^{n+1}\left(k-2 n-1+\frac{m}{2}\right)_{n} & j=2 n+1,\end{cases}
$$

which concludes the proof. 
As a consequence, we obtain the dimension of the space of discrete homogeneous monogenic polynomials of degree $k$. Indeed, from the Fischer decomposition (16) we obtain

$$
\operatorname{dim} \mathcal{M}_{k}=\operatorname{dim} \Pi_{k}-\operatorname{dim} \Pi_{k-1}
$$

while

This leads to the following theorem.

$$
\operatorname{dim} \Pi_{k}=\frac{(k+m-1) !}{k !(m-1) !} .
$$

Theorem 5.3. The space of discrete homogeneous monogenic polynomials of degree $k$ has dimension

$$
\operatorname{dim} \mathcal{M}_{k}=\frac{(k+m-1) !-k(k+m-2) !}{k !(m-1) !}=\frac{(k+m-2) !}{k !(m-2) !} .
$$

\section{ACKNOWLEDGEMENTS}

The work of the third author was (partially) supported by the Unidade de Investigação Matemática e Aplicações of Aveiro University. The fourth author gratefully acknowledges support by the research grant 31506208 of the Flemish Science Foundation (FWO-Krediet aan Navorsers).

\section{REFERENCES}

[1] F. Brackx, R. Delanghe, F. Sommen, Clifford Analysis, Research Notes in Mathematics 76, Pitman, Boston, MA, 1982. MR697564 (85j:30103)

[2] F. Brackx, H. De Schepper, F. Sommen, L. Van de Voorde, Discrete Clifford analysis: an overview, Cubo 11(1) (2009), 55-71. MR 2500184

[3] F. Brackx, H. De Schepper, F. Sommen, L. Van de Voorde, Discrete Clifford analysis: a germ of function theory. In: I. Sabadini, M. Shapiro, F. Sommen (eds.), Hypercomplex Analysis, Birkhäuser, 2009, 37-53.

[4] A.I. Bobenko, C. Mercat, Y. Suris, Linear and nonlinear theories of discrete analytic functions. Integrable structures and isomonodromic Green's function, J. reine und angew. Math. $\mathbf{5 8 3}$ (2005), 117-161. MR2146854 (2006m:37102)

[5] H. De Bie, F. Sommen, Fischer decompositions in superspace. In: Function spaces in complex and Clifford analysis, National University Publishers, Hanoi, 2008, 170-188. MR2405887 (2009f:58017)

[6] R. Delanghe, F. Sommen, V. Souček, Clifford algebra and spinor-valued functions, Kluwer Academic Publishers, Dordrecht, 1992. MR 1169463 (94d:30084)

[7] C.F. Dunkl, Y. Xu, Orthogonal polynomials of several variables, Encyclopedia of Mathematics and its Applications 81, Cambridge University Press, Cambridge, 2001. MR 1827871 (2002m:33001)

[8] D. Eelbode, Stirling numbers and spin-Euler polynomials, Exp. Math. 16(1) (2007), 55-66. MR2312977 (2008e:30060)

[9] N. Faustino, K. Gürlebeck, A. Hommel, U. Kähler, Difference potentials for the Navier-Stokes equations in unbounded domains, J. Diff. Eq. \& Appl. 12(6) (2006), 577-595. MR2240377 (2007g:76056)

[10] N. Faustino, U. Kähler, Fischer decomposition for difference Dirac operators, Adv. Appl. Cliff. Alg. 17(1) (2007), 37-58. MR.2303055 (2008a:30067)

[11] N. Faustino, U. Kähler, F. Sommen, Discrete Dirac operators in Clifford analysis, Adv. Appl. Cliff. Alg. 17(3) (2007), 451-467. MR2350591(2008i:30054)

[12] E. Forgy, U. Schreiber, Discrete differential geometry on causal graphs, preprint (2004), arXiv:math-ph/0407005v1.

[13] K. Gürlebeck, W. Sprößig, Quaternionic and Clifford Calculus for Engineers and Physicists, John Wiley \& Sons, Chichester, 1997.

[14] K. Gürlebeck, A. Hommel, On finite difference potentials and their applications in a discrete function theory, Math. Meth. Appl. Sci. 25 (2002), 1563-1576. MR1949515 (2004a:39037) 
[15] K. Gürlebeck, A. Hommel, On finite difference Dirac operators and their fundamental solutions, Adv. Appl. Cliff. Alg. 11 (2001), 89-106. MR2075345(2005h:30092)

[16] I. Kanamori, N. Kawamoto, Dirac-Kähler fermion from Clifford product with noncommutative differential form on a lattice, Int. J. Mod. Phys. A19 (2004), 695-736. MR2041732 (2005f:81118)

[17] H.R. Malonek, D. Peña Peña, F. Sommen, Fischer decomposition by inframonogenic functions, Cubo (to appear).

[18] B. Ørsted, P. Somberg, V. Souček, The Howe duality for the Dunkl version of the Dirac operator, Adv. Appl. Cliff. Alg. 19(2) (2009), 403-415. MR.2524678

[19] J. Vaz Jr., Clifford-like calculus over lattices, Adv. Appl. Cliff. Alg. 7(1) (1997), 37-70. MR1472066 (99c:30077)

Clifford Research Group, Department of Mathematical Analysis, Faculty of Engineering, Ghent University, Galglaan 2, 9000 Gent, Belgium

E-mail address: hdr@cage.UGent.be

Clifford Research Group, Department of Mathematical Analysis, Faculty of Engineering, Ghent University, Galglaan 2, 9000 Gent, Belgium

E-mail address: hds@cage.UGent.be

Departamento de Matemática, Universidade de Aveiro, Aveiro, 3810-193 Centro, PORTUGAL

E-mail address: ukaehler@ua.pt

Clifford Research Group, Department of Mathematical Analysis, Faculty of Sciences, Ghent University, Galglaan 2, 9000 Gent, Belgium

E-mail address: fs@cage.ugent.be 